\title{
TESTING THE CENTRAL ENGINES IN AGNS
}

\author{
N. YAMAZAKI, Y. TANIGUCHI, AND O. KABURAKI \\ Astronomical Institute, Tohoku University \\ Aramaki, Aoba, Sendai 980-77, JAPAN
}

\section{Theoretical Predictions}

It is very important to estimate the relationship between the central blackhole mass $M_{\mathrm{BH}}$ and bolometric luminosity $L_{\mathrm{bol}}$ of AGNs, because such a relation may be useful to judge the validity of various models for AGNs (Koratkar \& Gaskell 1991, hereafter KG91). The predictions of various models are summerized in Table 1 , where $B$ is the magnetic field around the central object.

TABLE 1. The model dependence of $M_{\mathrm{BH}}-L_{\mathrm{bol}}$ relationship

\begin{tabular}{ccc}
\hline Model & $M_{\mathrm{BH}}-L_{\mathrm{bol}}$ relationship & Reference \\
\hline Radiation-driven jet model & $L_{\mathrm{bol}} \propto M_{\mathrm{BH}}$ & Jaroszyński et al. 1980 \\
Accretion-driven jet model & $L_{\mathrm{bol}} \approx$ independent of $M_{\mathrm{BH}}$ & Pringle 1981, Kaburaki 1986 \\
Spinning black-hole model & $L_{\mathrm{bol}} \propto B^{2} M_{\mathrm{BH}}^{2}$ & Blandford \& Znajek 1977 \\
Grazer model & $L_{\mathrm{bol}} \propto B^{2} M_{\mathrm{BH}}^{2}$ & Kaburaki \& Taniguchi 1996 \\
& & Taniguchi \& Kaburaki 1996 \\
\hline
\end{tabular}

\section{Data}

Using the data obtained by Kaspi et al., we have newly derived the masses $\left(M_{\mathrm{BH}}\right)$ of central objects, PG0804+761 and PG0953+414 according to the method used in KG91. Adding these results to the 10 data of KG91 and omitting, instead, 5 original data points with very large errors, we obtain the mass-luminosity relation for AGNs. We find that $L_{\text {bol }}$ is proportional to $M_{\mathrm{BH}}^{2}$ rather than to $M_{\mathrm{BH}}^{1}$ (figure1). 


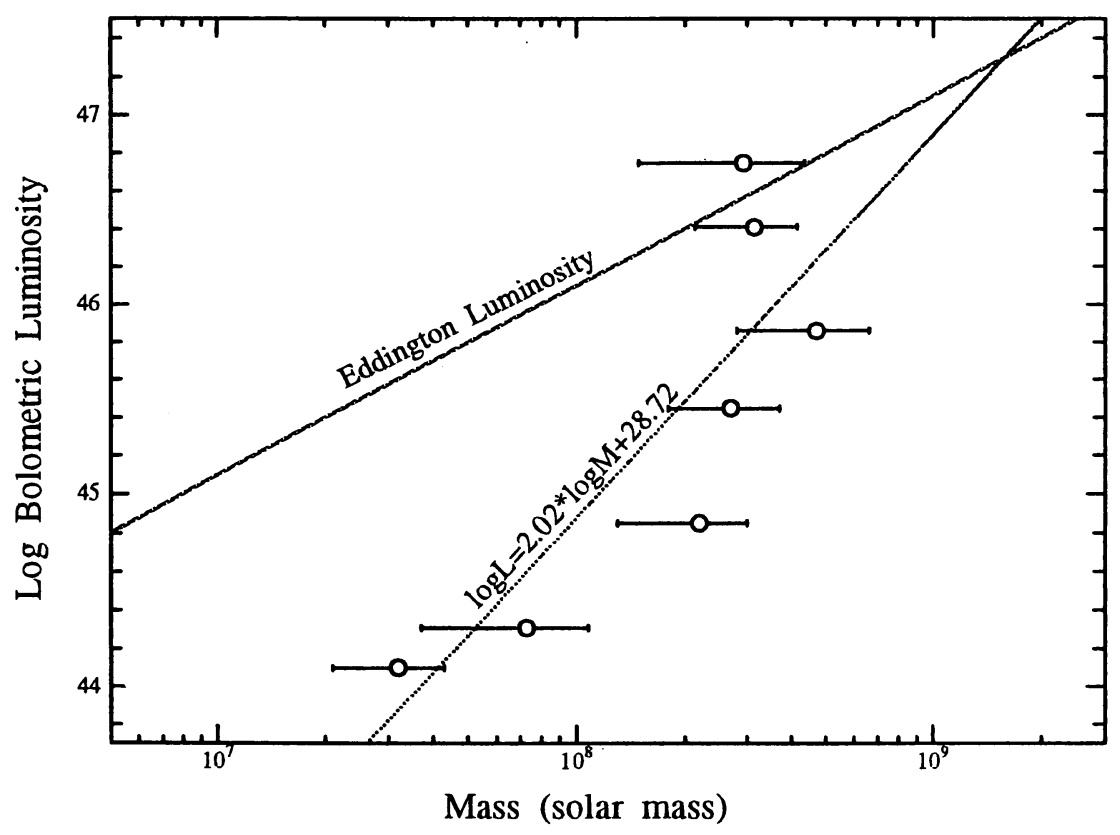

Figure 1. The relation between $M_{\mathrm{BH}}$ and $L_{\mathrm{bol}}$

\section{Conclusion}

Our tentative conclusion is that the most appropriate models of AGN engines are the spinning black hole model and the grazer model (Yamazaki et al. 1997). Radiation-driven jet model is not so appropriate but can not be rejected. The normal accretion-driven jet model is inappropriate.

\section{References}

Blandford, R. D., \& Znajek, R. L. (1977) MNRAS, 179, 433

Jaroszyński, M., Abramowicz, M. A., \& Paczyński, B. (1980) Acta Astronomica, 30, 1 Kaburaki, O. (1986) MNRAS, 220, 331

Kaburaki, O., and Taniguchi, Y. (1996) in Physics of Accretion Disks: Advection, Radiation, and Magnetic Field, edited by S. Kato et al. (Gordon and Breach Science Publishers), 327

Kaspi, S., Smith, P. S., Maoz, D., Netzer, H., \& Jannuzi, B. T. (1996) ApJ, 471, L75

Koratkar, A. P. \& Gaskell, C. M. (1991) ApJ, 370, L61 (KG91)

Padovani, P., \& Rafanelli, P. (1988) $A \& A$, 205, 53

Pringle, J. E. (1981) $A R A \& A, 19,137$

Taniguchi, Y., and Kaburaki, O. (1996) in The Physics of LINERs in View of Recent Observations, ASP Conf. Ser. 103, 227

Yamazaki, N., Taniguchi, Y., \& Kaburaki, O. (1997) ApJ, submitted 